\title{
Communicable Diseases Report, NSW, September and October 2010
}

\section{Communicable Diseases Branch NSW Department of Health}

For updated information, including data and facts on specific diseases, visit www.health.nsw.gov.au and click on Public Health and then Infectious Diseases. The communicable diseases site is available at: http://www.health.nsw.gov.au/publichealth/ infectious/index.asp.
Figure 2 and Tables 1 and 2 show reports of communicable diseases received through to the end of September and October 2010 in New South Wales (NSW).

\section{Enteric infections \\ Outbreaks of foodborne disease}

Eleven outbreaks of suspected foodborne disease were investigated in September and October 2010. In three of these outbreaks, stool specimens were collected from cases and tested. In one of these outbreaks in an aged care facility, stool specimens tested positive for Salmonella Infantis. No clear source of infection was identified although one chicken food sample collected from the facility tested positive for another Salmonella serogroup (S. Sophia). In another outbreak also in an aged care facility, stool specimens tested positive for Clostridium perfringens toxin but no clear source of infection was identified. Stool specimens from the third outbreak were positive for Salmonella Typhimurium (MLVA: 3-9-7-13523). While this outbreak was epidemiologically linked to consumption of pork rolls served at a bakery, none of the food samples that were subsequently collected from the bakery tested positive for Salmonella spp.

In two other outbreaks, food and/or environmental samples (e.g. microbiological swabs of food preparation areas) were taken but no pathogens were identified. In the remaining six outbreaks, none of the cases submitted a stool specimen for testing and a causative pathogen for the outbreak could not be identified. One of these outbreak investigations is ongoing.

\section{Outbreaks of gastroenteritis in institutional settings}

During September and October, 131 outbreaks of gastroenteritis in institutions were reported, affecting a total of 2182 people. Sixty-eight outbreaks occurred in aged care facilities, 39 in child care centres, 20 in hospitals, and one each in a military institution, school, pre-school and camp. All of these outbreaks appeared to have been caused by person-to-person spread of a viral illness. In 76 outbreaks $(58 \%)$ one or more stool specimens were collected from cases; in 39 of these outbreaks $(56 \%)$ norovirus was detected, and in $15(20 \%)$ rotavirus was detected. Stool specimen test results for several outbreaks are pending. Viral gastroenteritis tends to peak in winter with around 15 outbreaks each week; over the past 5 years in September and October the average number of outbreaks has been 124 .

\section{Gastroenteritis in the community}

The number of patients presenting with gastrointestinal illness who presented to 56 of the state's largest emergency departments in NSW remained steady and was just below the usual range for this time of year (Figure 1).

\section{Respiratory and other infections Influenza}

During September and October influenza activity was low in NSW, as measured by the number of patients who presented to 56 of the state's largest emergency departments with influenza-like illness and the number of patients who tested positive for influenza at diagnostic laboratories.

- There were 344 emergency department presentations of patients with influenza-like illness (1.8 per 1000 presentations) for September and 287 presentations (1.9 per 1000 presentations) for October.

- There were 306 cases of laboratory-confirmed influenza (including 241 of pandemic (H1N1) 2009) reported in September and 160 (including 125 of pandemic (H1N1) 2009) in October.

- A total of 26 patients with laboratory-confirmed influenza were admitted to hospital intensive care units; 14 in September and 12 in October.

For a more detailed report on influenza disease activity in NSW see: http://www.health.nsw.gov.au/PublicHealth/ Infectious/influenza_reports.asp.

\section{Vaccine-preventable diseases Measles}

Eight cases of measles were reported in September and October. All three confirmed cases were associated with a 
previously reported measles outbreak in the NSW North Coast Area, ${ }^{1}$ and all had epidemiological links with a secondary case, an inmate at a prison in the North Coast Area.

Serological surveys indicate that measles immunity is high (98\%) in people born before 1968 due to exposure to wild measles, but lower (89\%) in people born from 1974 to 1980 due to less frequent exposure to wild virus and lower rates of vaccine uptake. ${ }^{2}$ Vaccines which protect against measles are now routinely given to infants at 12 months and at 4 years, and this provides long-lasting immunity in $99 \%$ of recipients. These recent outbreaks highlight the importance of completing measles immunisation, particularly prior to overseas travel.

\section{Meningococcal disease}

Eighteen cases of meningococcal disease were reported in NSW in September and October 2010 (14 cases were reported in the same period in 2009). The ages of the affected people ranged from 0 to 83 years (six cases were children aged less than 5 years). Twelve cases were caused by serogroup B, one case was caused by serogroup C (in an unvaccinated adult), and one case was caused by serogroup W135. For four cases the serogroup was unknown.

To date this year, 63 cases of meningococcal disease have been reported in NSW (including five deaths) compared to 80 cases for the same period in 2009 (including four deaths).

A free vaccine for serogroup $\mathrm{C}$ meningococcal disease is available for infants at 12 months of age. Consequently, serogroup $\mathrm{C}$ meningococcal disease is now mainly seen in adults and in unimmunised children. In NSW this year, $82 \%$ of cases of meningococcal disease (where the serogroup was known) have been caused by serogroup B, for which there is no vaccine.

\section{Pertussis (whooping cough)}

During September and October, 2108 cases of pertussis were reported in NSW. Over 20000 cases of whooping cough were reported during 2008 and 2009. Case reports declined to a low in April this year (with 314 cases reported), but have since increased, with 1201 cases reported in October. To date, 5423 cases have been reported in NSW compared with 11370 for the same period in 2009.

A free vaccine is available for infants at 2, 4 and 6 months (the first dose can be given as early as 6 weeks of age) with a booster dose at 4 years (which can be given from 3 years and 6 months of age). Immunisation reduces the risk of infection, however the vaccine does not provide lifelong protection and re-infection can occur. Because pertussis immunity wanes over time, many older children and adults are susceptible to infection and can be the source of new infections in infants. For a limited time, free pertussis (dTpa) vaccine is available for all new parents, grandparents and any other adults who will regularly care for infants less than 12 months of age. Free vaccine is also provided to Year 7 and Year 10 students as part of the NSW School-based Vaccination Program.

\section{Haemophilus influenzae type $b$ invasive infection}

One case of Haemophilus influenzae type $\mathrm{b}$ invasive infection (Hib) was reported in a fully vaccinated, nonindigenous 3-year old child in NSW in September. For the same period in 2009, there were no cases of Hib reported in NSW in any age group.

\section{Tuberculosis program}

In October 2010, a case of extensively drug-resistant tuberculosis (XDR-TB) was identified in a NSW resident who had been treated for tuberculosis overseas. XDR-TB is a relatively rare event globally and this is only the second case identified in Australia. Tuberculosis can usually be

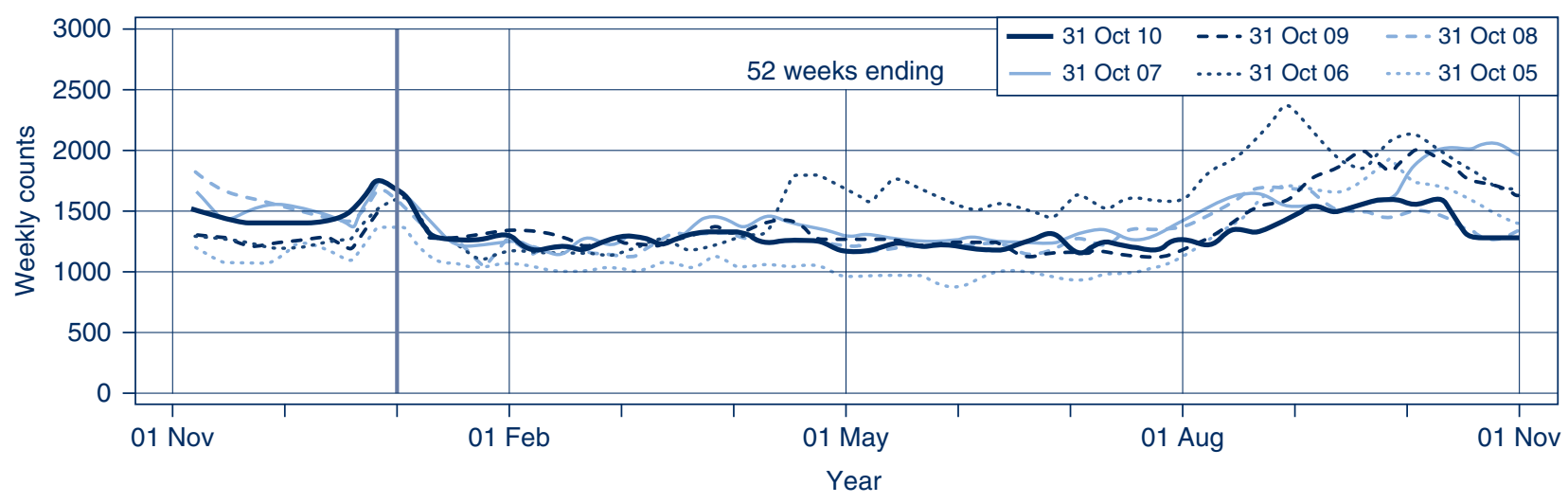

Figure 1. Total weekly counts of emergency department visits for gastrointestinal illness, for the 12 months to 30 October 2010 (thick line), compared with each of the previous 5 years (thin lines) (includes data from 56 of the largest emergency departments in NSW).

Source: Public Health Real-time Emergency Department Surveillance System, NSW Department of Health. 
treated with a course of four standard, or first-line, antituberculosis drugs. If treatment with these drugs is mismanaged or interrupted, multidrug-resistant tuberculosis (MDR-TB) can develop. When these second-line drugs are also mismanaged or interrupted, XDR-TB can develop. As XDR-TB is resistant to first-line and second-line drugs, patients are left with treatment options that are much less effective.

Further information on XDR-TB can be obtained from the World Health Organization (http://www.who.int/tb/ challenges/xdr/faqs/en/index.html).

On identification of a case of MDR-TB or XDR-TB, NSW Health convenes an Expert Panel to develop treatment, management and public health strategies to minimise the potential for further spread of the disease and to promote an effective cure for the individual. This process is described under the NSW policy directive PD2005_159: Tuberculosis - Management of Multi Drug Resistant Tuberculosis in NSW (http://www.health.nsw.gov.au/policies/PD/2005/ PD2005_159.html).

The management of this XDR-TB case was reviewed by an Expert Panel and the case is reported to be responding well to treatment.

\section{Sexually transmissible infections Human Immunodeficiency Virus (HIV)}

The numbers of newly diagnosed HIV cases notified to NSW Health in 2009 have recently been finalised. In 2009, 328 people newly diagnosed with HIV infections were notified in NSW, which was similar to the number in 2008 (324).

For newly diagnosed cases reported in 2009 the median age at diagnosis was 38 years and a total of $289(88 \%)$ were male. The majority of cases were reported to be homosexually acquired (235); for the other cases acquisition was identified as heterosexual (77), injecting drug use (10) and through other or unknown sources (6).

Overall, the number of people newly diagnosed with HIV infection in NSW remained stable in 2009. Promoting safe sex practices and regular testing among men who have sex with men remains important as this was the most commonly reported exposure in 2008.

Further information is available in the document 'Summary of HIV in 2009' available at: http://www.health.nsw. gov.au/PublicHealth/Infectious/a-Z.asp.

\section{Syphilis}

A total of 295 cases of infectious syphilis were reported in NSW in the 10 months to the end of October 2010. This is a decrease in the number of cases of syphilis notified to NSW Health compared with the number notified in 2009 at the same time last year (444).

Syphilis is a highly infectious sexually transmitted disease that is spread through vaginal, anal or oral sex through skin-to-skin contact. Syphilis is highly contagious during the primary and secondary stages when the sore or rash is present. Those most at risk include men who have sex with men, people with HIV/AIDS and people living in Aboriginal communities that are remote or have poor access to health care services.

In April 2010 the NSW public health response to syphilis notifications changed to provide support to doctors in diagnosing and managing syphilis. Public health units now refer notifications in men aged less than 60 years and women aged less than 45 years to the local sexual health clinic in instances where these patients are being managed by doctors who are not known to have experience in the management of syphilis. Sexual health clinic staff offer these doctors assistance to complete the notification form. The decrease in notifications began prior to the implementation of this change. A review of the new procedure is to be conducted.

\section{References}

1. Communicable Diseases Report, NSW, July and August 2010. N S W Public Health Bull 2010; 21(9-10): 250-5.

2. Gidding HF, Gilbert GL. Measles immunity in young Australian adults. Commun Dis Intell 2001; 25: 133-6. 
Figure 2. Reports of selected communicable diseases, NSW, January 2004 to October 2010, by month of onset. Preliminary data: case counts in recent months may increase because of reporting delays.

Laboratory-confirmed cases only, except for measles, meningococcal disease and pertussis. BFV, Barmah Forest virus infection; RRV, Ross River virus infections; lab conf, laboratory confirmed; Men $\mathrm{Gp} C$ and $\mathrm{Gp} B$, meningococcal disease due to serogroup $C$ and serogroup $B$ infection; other/unk, other or unknown serogroups.

NB: Multiple series in graphs are stacked, except gastroenteritis outbreaks.

NB: Outbreaks are more likely to be reported by nursing homes \& hospitals than by other institutions.

\begin{tabular}{|lr|}
\hline \multicolumn{2}{|c|}{ NSW Population } \\
Male & $50 \%$ \\
$<5 y$ & $7 \%$ \\
$5-24$ y & $27 \%$ \\
$25-64$ y & $53 \%$ \\
$65+y$ & $13 \%$ \\
Rural & $46 \%$ \\
\hline
\end{tabular}

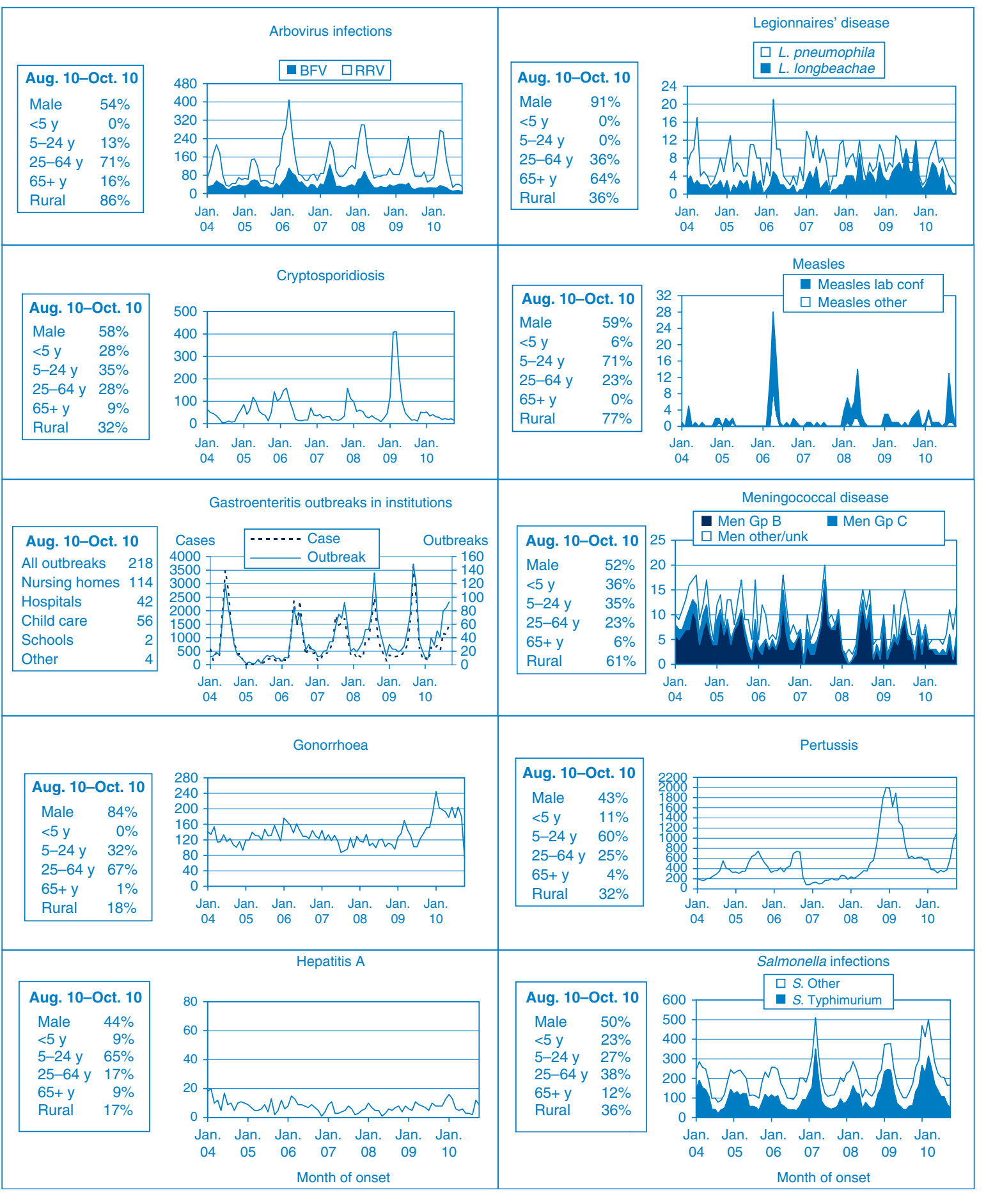




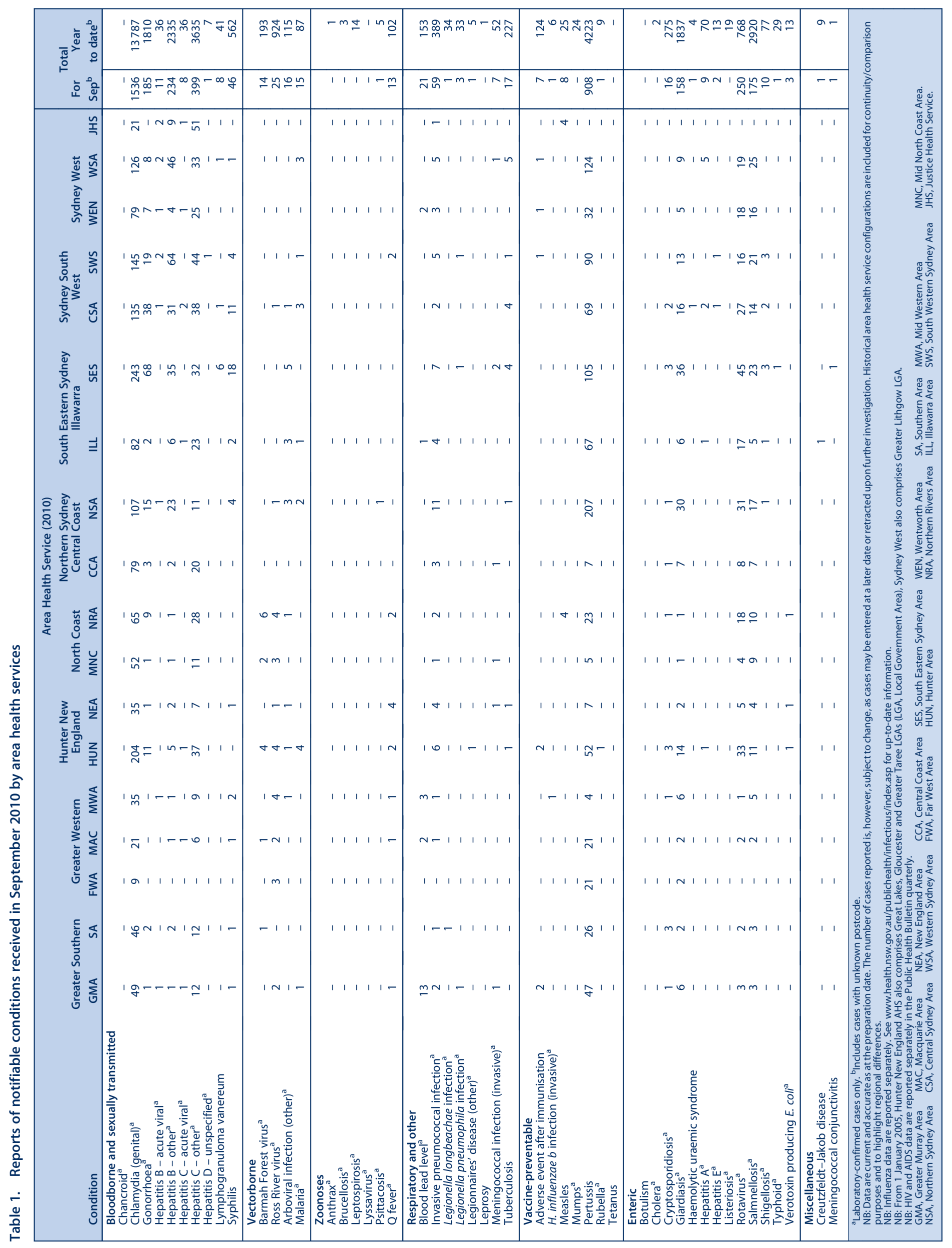




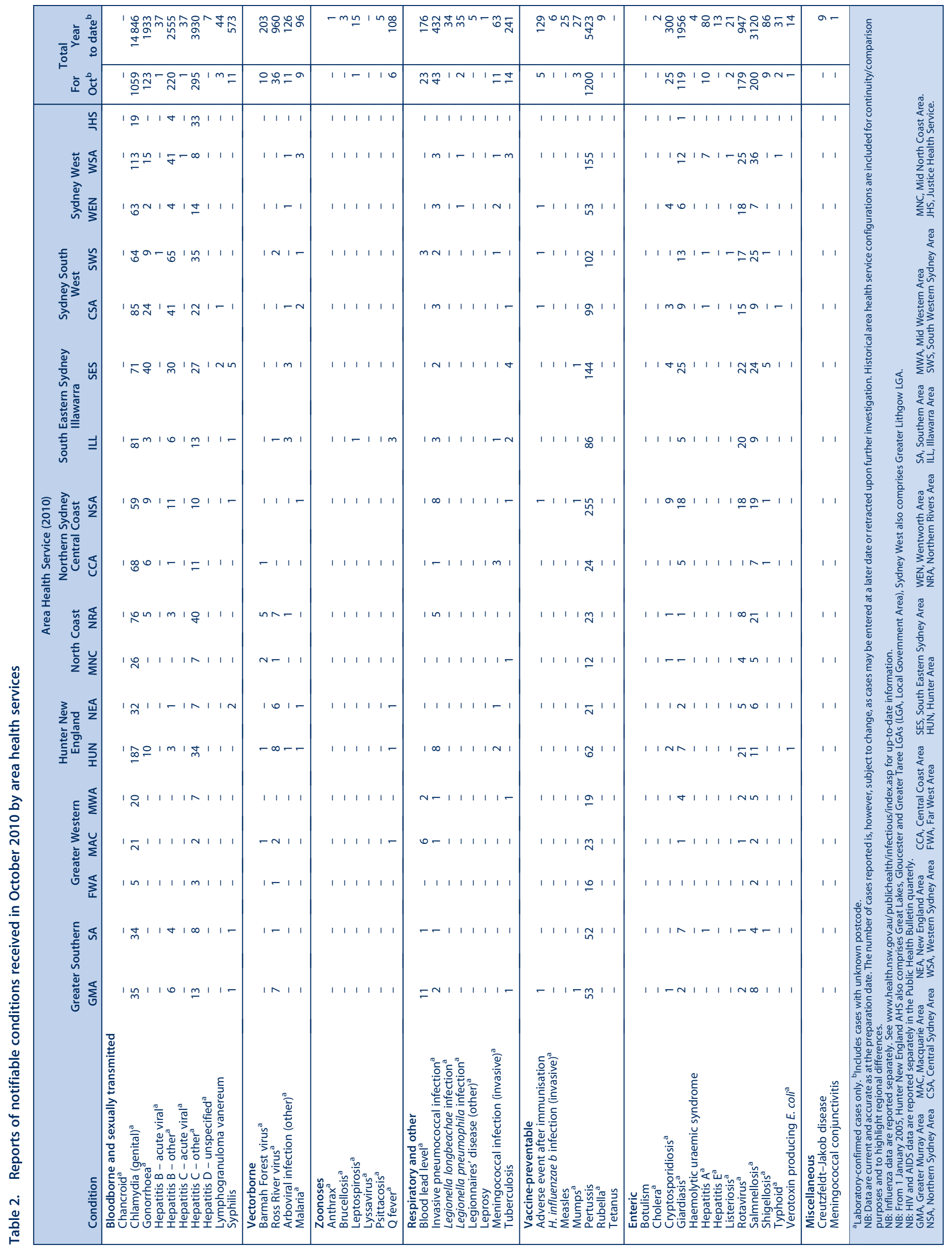

